\title{
Phase separation and electron pairing in repulsive Hubbard clusters
}

\author{
G. W. Fernando,,${ }^{1,4}$ A. N. Kocharian, ${ }^{2,5}$ K. Palandage, ${ }^{1}$ Tun Wang, ${ }^{1}$ and J. W. Davenport ${ }^{3}$ \\ ${ }^{1}$ U-46, Physics Department, University of Connecticut, Storrs, Connecticut 06269, USA \\ ${ }^{2}$ Physics Department, California State University, Northridge, California 91330, USA \\ ${ }^{3}$ Computational Science Center, Brookhaven National Laboratory, Upton, New York 11973, USA \\ ${ }^{4}$ Institute of Fundamental Studies, Hantana Road, Kandy, Sri Lanka \\ ${ }^{5}$ Pierce College, Woodland Hills, California 91371, USA \\ (Received 14 December 2006; published 28 February 2007)
}

\begin{abstract}
Exact thermal studies of small (4-site and 8-site) Hubbard clusters with local electron repulsion yield intriguing insight into phase separation, charge-spin separation, pseudogaps, condensation, and, in particular, pairing fluctuations away from half-filling. These exact calculations, carried out in canonical and grand canonical ensembles, monitoring variations in temperature $T$ and magnetic field $h$, show rich phase diagrams in a $T-\mu$ space consisting of pairing fluctuations and signatures of condensation. Corresponding electron pairing instabilities are seen when the on-site Coulomb interaction $U$ is smaller than a critical value $U_{c}(T)$ and they point to a possible electron pairing mechanism. The specific heat, magnetization, charge pairing, and spin pairing provide strong support for the existence of competing (paired and unpaired) phases near optimal doping in these clusters, and numerous similarities with experiments on high $T_{c}$ superconductors are pointed out. In addition, these ideas may be linked to superconducting carbon nanotubes where a purely electronic mechanism could be responsible for superconductivity.
\end{abstract}

DOI: 10.1103/PhysRevB.75.085109

PACS number(s): 71.10.Fd, 65.80.+n, 71.27. $+\mathrm{a}, 73.22 .-\mathrm{f}$

\section{INTRODUCTION}

Nanoscience has become one of the frontiers of modern physics and has generated tremendous interest among both the scientific and nonscientific communities. Nanoclusters (such as quantum dots, rods, grains) are becoming extremely useful in medical and engineering applications. The ability to synthesize small clusters containing a few atoms provides a unique opportunity to examine and tune fundamental physical and chemical properties. Hence, it is important to conduct theoretical studies of such clusters, and this work reports a number of surprising results based on the methodology discussed in our previous work. ${ }^{1,2}$

The significance of the present study is twofold: First, although numerous properties, including eigenvalues and susceptibilities, of Hubbard clusters have been calculated ${ }^{3-11}$ previously, many open questions remain with regard to microscopic origins of charge-spin separation, pseudogap behavior, and various scenarios of possible pairings at low temperature. Apparently, the above studies did not search for transitions, as we have done here, over an extended parameter space that includes variations in chemical potential, magnetic field, Coulomb repulsion, and temperature. Insight into the properties of Hubbard clusters is gained by monitoring weak singularities in susceptibilities over this extended parameter space. ${ }^{12}$ It is our hope that this work would motivate further studies of electronically driven mechanisms for pairing, etc., in correlated nanosystems.

Second, our results may be relevant to doped fullerenes, ${ }^{13}$ superconducting carbon nanotubes $(\mathrm{CNs}),{ }^{14}$ organic superconductors, ${ }^{15}$ as well as high-temperature superconductors (HTSCs) in the search for a purely electronic mechanism for superconductivity. It is imperative that such similarities be carefully examined and understood, given the low dimensionality of CNs and the inhomogeneities observed in the HTSCs. ${ }^{16,17}$ In addition, while one-dimensional theorems preclude long-range order, it is still an open question whether finite clusters can retain ground-state, short-range correlations at finite temperature. ${ }^{2,18}$

Since the discovery of HTSCs, there has been an intense debate about a possible electron (or hole) pairing mechanism. Early on, Anderson ${ }^{19}$ suggested that the large positive on-site Coulomb interaction in the Hubbard model should contain the key to some of the perplexing physics observed in the HTSCs. The ground state of the model, in different geometries, has also been suggested as providing a mechanism for electron pairing in fullerenes and certain mesoscopic structures due to intramolecular correlation effects at moderate $U .{ }^{16}$ Our recent work ${ }^{1}$ indicates that an ensemble of Hubbard clusters, when connected to a particle reservoir and a thermal bath, possesses a vivid variety of interesting thermal and ground-state properties. These inferences were drawn by carrying out exact diagonalizations of the manybody Hamiltonian and using these eigenvalues in a statistical ensemble to study ground-state transitions and thermal crossovers by monitoring susceptibilities, i.e., fluctuations. Such ensemble averages have been demonstrated to be relevant for nanoclusters. ${ }^{20}$ Although there have been numerous studies of phase separation and similar phenomena in Hubbard systems, ${ }^{16,21}$ in our opinion, thermal properties and phase instabilities of small Hubbard clusters at an arbitrary filling have not been fully explored.

\section{METHODOLOGY AND KEY RESULTS}

The single orbital Hubbard Hamiltonian,

$$
H=-t \sum_{\langle i j, \sigma\rangle} c_{i \sigma}^{+} c_{j \sigma}+\sum_{i} U n_{i \uparrow} n_{i \downarrow},
$$

with hopping $t$ and on-site interaction $U$, has been used in this work with periodic boundary conditions. In order to 
study thermal properties, the many-body eigenvalues of the Hubbard clusters are combined with the grand canonical potential $\Omega_{U}$ for interacting electrons with

$$
\Omega_{U}=-T \ln \sum_{n \leqslant N_{H}} e^{-\left(E_{n}-\mu N_{n}-h s_{n}^{z}\right) / T},
$$

where $N_{n}$ and $s_{n}^{z}$ are the number of particles and the projection of spin in the $n$th state, respectively. The grand partition function $Z$ (where the number of electrons $N$ and the projection of spin $s^{z}$ can fluctuate) and its derivatives are calculated exactly without taking the thermodynamic limit. The response functions related to electron or hole doping (i.e., chemical potential $\mu$ ) or magnetic field $h$ demonstrate clearly observable prominent peaks, paving the way for strict definitions of Mott-Hubbard (MH), antiferromagnetic, spin pseudogaps and related crossover temperatures. ${ }^{1,2}$ Unless otherwise stated, all the energies reported here are measured in units of $t$ (i.e., $t$ has been set to 1 in most of the work that follows). For a complete description of the method, see Ref. 1.

We have already shown that in such finite systems, one can define the gap parameters for various transitions and identify corresponding phase boundaries by monitoring maxima and minima in charge and spin susceptibilities. ${ }^{1}$ As synthesis techniques improve at a rapid rate, it has become possible to synthesize isolated clusters, and hence it is clear that we need not always look at the thermodynamic limit. Finite, mesoscopic structures in suitable topological forms will be realistic enough to synthesize [such as the CNs (Ref. 14)] and extract fascinating physics. Also, since the HTSCs are known to consist of (stripes and possibly other) inhomogeneities, ${ }^{17}$ it is possible that these cluster studies may be able to capture some of the essential physics of the HTSCs. The following is a list of key results from our exact (4-site and 8-site) Hubbard cluster studies:

(1) phase diagrams in a temperature-chemical potential (doping) plane and the presence of a multitude of fascinating phases, including Mott-Hubbard-like paramagnetic and antiferromagnetic phases; ${ }^{1}$

(2) vanishing of a charge gap at a critical set of parameters and thereby providing an effective attraction, leading to onset of electron pairing at a critical temperature $T_{c}^{P}$;

(3) spin pairing at a lower temperature $\left(T_{s}^{P}\right)$ and hence the formation of rigidly bound spin pairs in a narrow, critical region of doping;

(4) low-temperature specific heat peak, reminiscent of the experimental, low-temperature specific heat behavior in the HTSCs; ${ }^{22}$

(5) temperature vs $U$ phase diagram, indicating the pressure effect on the superconducting transition temperature similar to reported results of recent experiments; $; 3,15,23$

(6) the presence of a dormant magnetic state, lurking in the above narrow, critical region of doping, that could be stabilized by either applying a magnetic field, going above the spin pairing temperature, or changing the chemical potential, similar to what is observed in a recent, notable experiment; ${ }^{24}$
(7) the opening of a pseudogap above the pairing temperature, similar to what is seen in NMR experiments, in both hole and electron doped cuprates, ${ }^{25}$ and

(8) larger clusters with different topologies and higher dimensionality, illustrating how the above properties get scaled with size.

\section{CHARGE AND SPIN PAIRINGS}

Our exact studies of 4-site clusters indicate a net electron attraction leading to the formation of bound electron pairs and possible condensation at finite temperature for $U$ $<U_{c}(T) .{ }^{1,2}$ This pairing mechanism in the 4-site cluster, at $1 / 8$ (optimal) hole doping $(\langle N\rangle \approx 3)$ away from half-filling, exists when the on-site Coulomb interaction $U$ is less than an analytically obtained critical value, $U_{c}(T=0)=4.584$ (in units of the hopping parameter $t$ ). This critical value, reported in Ref. 2, is temperature dependent and can be associated with an energy gap (order parameter) which becomes negative below $U_{c}(T)$, implying that it is more energetically favorable to have a bound pair of electrons (or holes) compared to two unpaired ones at an optimal chemical potential (or doping level) $\mu=\mu_{P}=0.658$. Above this critical value $U_{c}(T)$, there is a Mott-Hubbard-like gap that exists when the average particle number $\langle N\rangle \approx 3$; this gap decreases monotonically as $U$ decreases and vanishes at $U_{c}(T)$. The vanishing of the gap indicates the onset of pair formation. There is an interval (width) around $\mu_{P}$, where the pairing phase competes with a phase (having a high magnetic susceptibility) that suppresses pairing at "moderate" temperatures.

An enlarged view of the $T$ - $\mu$ phase diagram for the planar 4-site cluster near $\mu_{P}$ is shown in Fig. 1. This exact phase diagram (at $U=4)$ in the vicinity of the optimally doped $(N$ $\approx 3$ ) regime has been constructed using the ideas described in the text and in Ref. 1. The electron pairing temperature $T_{c}^{P}$ identifies the onset of charge pairing. As temperature is further lowered, spin pairs begin to form at $T_{s}^{P}$. At this temperature (with zero magnetic field), spin susceptibilities become very weak, indicating the disappearance of the $\langle N\rangle \approx 3$ states. Below this spin pairing temperature $T_{s}^{P}$, only paired states are observed to exist having a certain rigidity, so that a nonzero magnetic field or a finite temperature is required to break the pairs. From a detailed analysis, it becomes evident that the system is on the verge of an instability, the paired phase competing with a phase that suppresses pairing which has a high, zero-field magnetic susceptibility. As the temperature is lowered, the number of $\langle N\rangle \approx 3$ (unpaired) clusters begins to decrease, while a mixture of (paired) $\langle N\rangle \approx 2$ and $\langle N\rangle \approx 4$ clusters appears. Interestingly, the critical doping $\mu_{P}$ (which corresponds to a filling factor of $1 / 8$ hole doping away from half-filling), where the above pairing fluctuations take place when $U<U_{c}(T)$, is close to the doping level near which numerous intriguing properties have been observed in the hole-doped HTSCs.

Specific-heat calculations (Fig. 2), associated with energy fluctuations, also provide further support for an electronic phase change at low temperature. As seen in this figure, there 


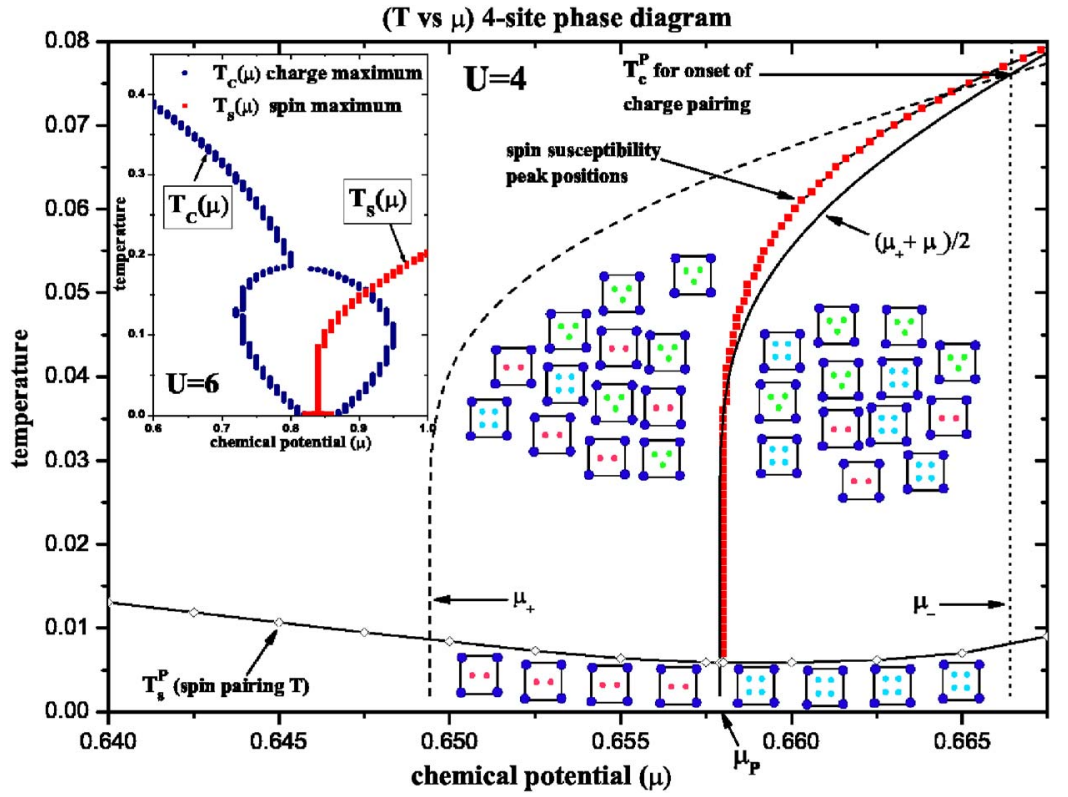

FIG. 1. (Color online) The $T-\mu$ phase diagram near $\mu_{P}=0.658(\langle N\rangle \approx 3)$ at $U=4$ for the 4 -site cluster. The inset shows a corresponding section (at a different scale) of the $T-\mu$ phase diagram for $U=6$. For $U=4$, note how the paired states condense at low temperature $\left(T_{s}^{P} \leqslant 100 \mathrm{~K}\right.$ if $t=1 \mathrm{eV}$ ) with a nonzero pair binding energy, while at higher temperatures, unpaired states begin to appear. This picture supports the idea that there is an inhomogeneous, electronic phase separation here. When $U$ is higher than $U_{c}(0)$ $=4.584$ (see inset for $U=6$ ), these inhomogeneities disappear and a Mott-Hubbard-like stable, paramagnetic, insulating region results around optimal doping. Note that there is charge-spin separation at $U=6$ in this gapped, insulating region [charge and spin susceptibility peaks are denoted by $T_{c}(\mu)$ and $T_{s}(\mu)$ ], as discussed in Ref. 1 . is a well separated, low-temperature peak at $\mu_{P}=0.658$ (around $40 \mathrm{~K}$, if the hopping parameter is set to $1 \mathrm{eV}$ and $U$ to $4 \mathrm{eV}$ ). This peak, which shifts to higher temperatures when the doping level is different from the critical doping level, is due to fluctuations between paired states $(\langle N\rangle=2$ and $\langle N\rangle=4)$. This low-temperature peak is in agreement with specific-heat experiments carried out for the HTSCs, ${ }^{22}$ and is a manifestation of the near degeneracy of the states in the neighborhood of critical doping $\mu_{P}$ and onset of condensation.

\section{IV. $T$ - $U$ PHASE DIAGRAM AND PRESSURE EFFECTS}

The $T$ - $U$ phase diagram in Fig. 3 illustrates the effects of the Coulomb repulsion $U$ on the possible binding of electron charge and spin in the 4-site cluster. The charge and spin

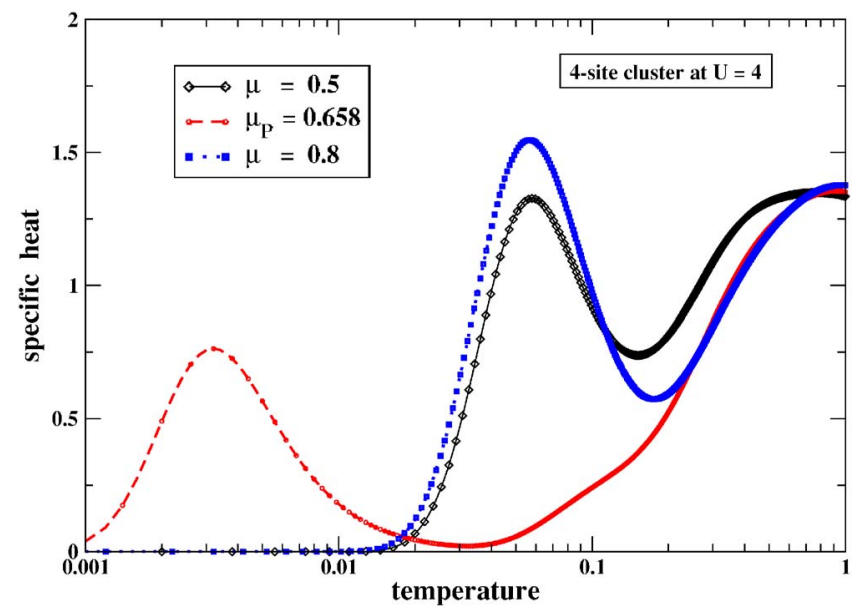

FIG. 2. (Color online) Specific heat vs temperature at $U=4$ calculated in the grand canonical ensemble for the 4-site cluster at several doping values near the critical doping, $\mu_{P} \approx 0.658$. Note how the low-temperature peak shifts to higher temperatures when the doping is changed from its critical value. pairing phase boundaries, $T_{c}^{P}(U)$ and $T_{s}^{P}(U)$, shown in Fig. 3 are constructed by monitoring the vanishing of the charge and the spin gaps ${ }^{1}$ near optimal doping as functions of $T$ and $U$. For temperatures $T_{s}^{P}(U) \leqslant T \leqslant T_{c}^{P}(U)$, there are a bound charge $2 e$ and decoupled spins. Below the lower curve, when $T \leqslant T_{s}^{P}(U)$, the spin degrees are also bounded and a finite applied magnetic field is needed to break them. ${ }^{1,8}$ In Fig. 3 for $U>U_{c}$, notice the existence of electron-hole pairing in a Mott-Hubbard-like insulating region [below temperature

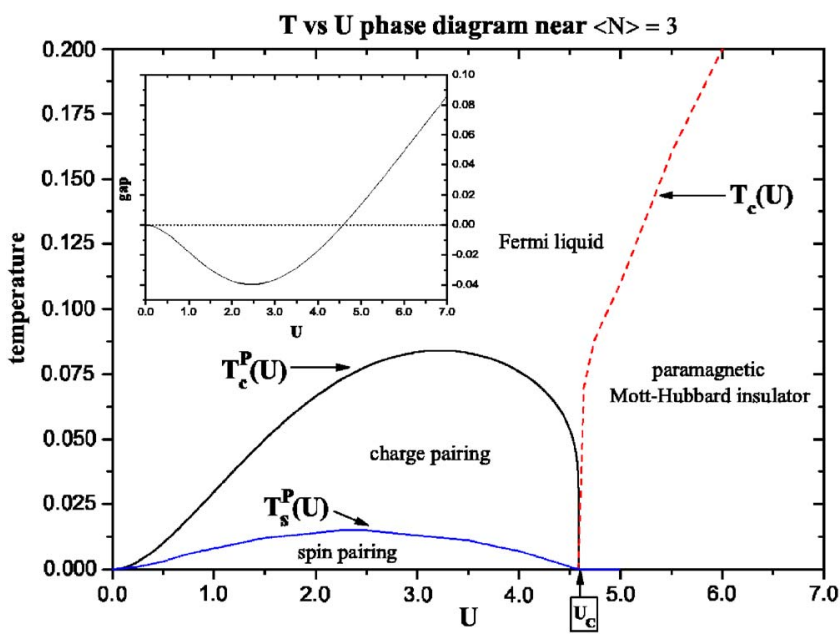

FIG. 3. (Color online) $T$ vs $U$ phase diagram for the optimally doped 4-site clusters, based on our exact calculations. $T_{c}^{P}(U)$ denotes the temperature at which the charge gap (see text) vanishes. Note that the pressure effects can be related to the behavior of $T_{s}^{P}(U)$, below which the spins are paired. Increasing the pressure is equivalent to decreasing $U$, while $t$ is held constant at $t=1$. The inset shows the charge gap as a function of $U$ at zero temperature. A negative charge gap implies charge pairing. Note that when $U$ $>U_{c}$, there is neither charge nor spin pairing. Instead, a MH-like insulating phase, with a positive charge gap at low temperature, is found near $\langle N\rangle \approx 3$; its phase boundary $\left[T_{c}(U)\right]$ is denoted by the dashed line. 


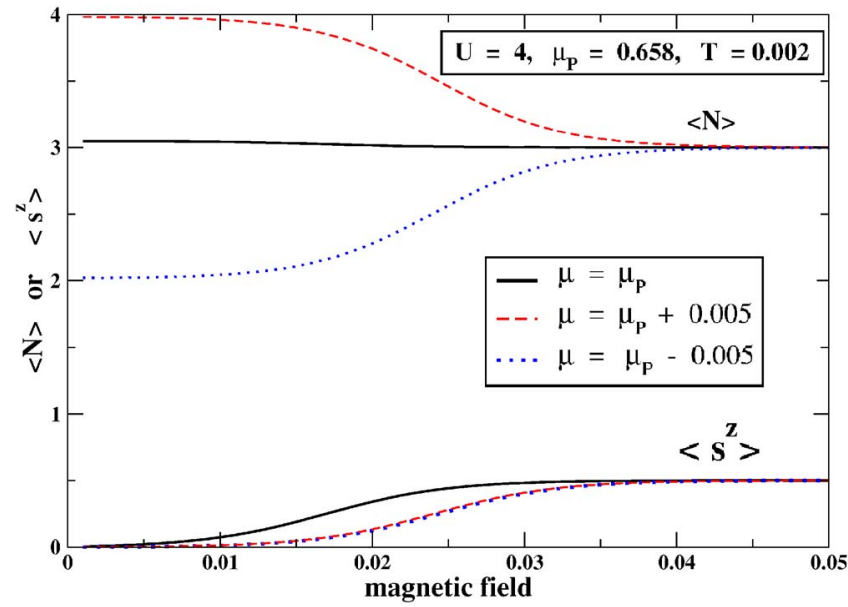

FIG. 4. (Color online) Variation of electron number $\langle N\rangle$ and magnetization $\left\langle s^{z}\right\rangle$ as a function of external magnetic field for several values close to critical doping $\mu_{P}=0.658$ at $T=0.002$ and $U$ $=4$ for the 4 -site cluster. Note how the $\langle N\rangle=3$ clusters get stabilized in a nonzero magnetic field at low temperature. These results are reminiscent of the recent observation of a magnetic state near optimal doping in hole-doped La cuprates (Ref. 24).

$T_{c}(U)$ denoted by the dashed line] away from half-filling. ${ }^{12}$ The inset in Fig. 3 shows the variation of the charge gap, $E(2)+E(4)-2 E(3)$, as a function of $U$, where $E(N)$ refers to the canonical energy for $N$ electrons at $T=0$. When this gap is negative, pairing is favored, as discussed in Ref. 1.

Figure 3 can also be used to understand pressure related effects, noting that increasing the pressure is equivalent to decreasing $U / t$. Hence, the increase of $T_{s}^{P}(U)$ with decreasing $U$, while holding $t$ constant at 1 (in Fig. 3) when 2.5 $\leqslant U \leqslant U_{c}$, reproduces the superconducting transition temperature (STT) vs pressure $p$ behavior in organic superconductors ${ }^{15}$ and optimally and nearly optimally doped HTSC materials ${ }^{23}$ indicating a significant role of pair binding in enhancing the STT. Notice that at small enough $U$, $T_{s}^{P}(U)$ decreases with decreasing $U$ (i.e., under increasing pressure), as shown in Fig. 3. This might explain why the pressure strongly depresses the STT across some families of alkali doped fullerene as well as organic superconductors. ${ }^{13}$

\section{UNPAIRED, DORMANT MAGNETIC STATE}

Another intriguing fact emerging from the exact thermal studies of the 4-site clusters is the existence of a dormant magnetic state (unpaired states with $\langle N\rangle=3$ ) with a high magnetic susceptibility. At rather low temperature $T \leqslant T_{s}^{P}$, this state is dormant. However, a small magnetic field or a change in chemical potential can stabilize it over the paired states $\langle N\rangle \approx 2,4$, as seen in Fig. 4 and the calculated grand canonical probabilities (not shown). The magnetization curves indicate the fact that the $\langle N\rangle \approx 3$ unpaired states are easier to magnetize with an infinitesimal field. The variation of the magnetic field mimics the doping to some extent here. Small changes in doping (at zero field) can also switch the system from one state to another with a different $\langle N\rangle$. These

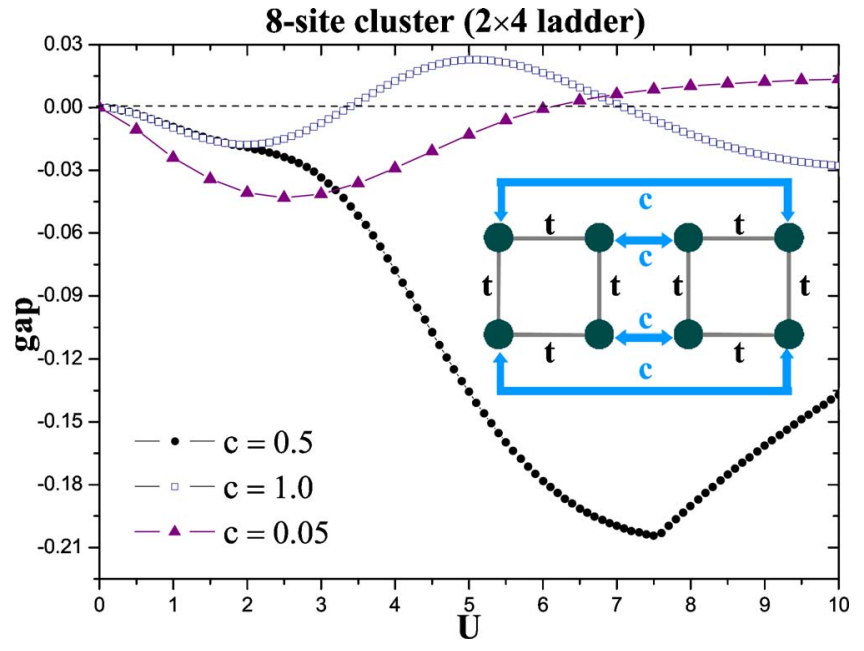

FIG. 5. (Color online) Charge gaps for the $2 \times 4$ cluster at $T$ $=0$ for various couplings $\mathbf{c}$ between the squares. The doping level is one electron off half-filling and the couplings $t$ within the squares are set to 1 , as indicated. There is an effective electron-electron attraction in the negative charge gap regions.

may be compared to some recent experimental results reported in Ref. 24, where a magnetic (and nonsuperconducting) state has been observed near $1 / 8$ hole doping in $\mathrm{La}_{2-x} \mathrm{Sr}_{x} \mathrm{CuO}_{4+y}$. This system is said to be on the verge of an instability, surprisingly similar to the phase-space region at $\langle N\rangle \approx 3$ (i.e., near optimal doping away from half-fillingsee Figs. 1 and 4) at low temperature. The physics behind this can be directly tied to the correlation driven instability near optimal doping when $U \leqslant U_{c}(T)$.

\section{LINKED 4-SITE CLUSTERS}

In order to monitor size effects, it is important to carry out numerical calculations for clusters with different topologies and sizes. ${ }^{7} 16$ Figure 5 illustrates one such set of our preliminary calculations of charge gaps carried out for an 8-site cluster ( $2 \times 4$ ladder), where the hopping term or coupling $c$ between the two squares was allowed to be different from the coupling within a given square. The pairing fluctuations that are seen for the 4-site cluster exist even for these ladders near half-filling, at optimal doping $(\langle N\rangle \approx 7)$, and most of the trends observed for the 4-site clusters, such as the MH-like charge gaps and vanishing of such gaps at critical $U$ values, remain valid here. The fluctuations that occur here at optimal doping are among the states with $\langle N\rangle \approx 6,7$, and 8 electrons. Clearly, the dormant magnetic state corresponds to $\langle N\rangle \approx 7$. Thermal and quantum fluctuations in the density of holes between the clusters [for $\left.U<U_{c}(0)\right]$ make it energetically more favorable to form pairs. In this case, snapshots of the system at relatively low temperatures and at a critical doping level (such as $\mu_{P}$ in Fig. 1) would reveal phase separation and equal probabilities of finding hole-rich or hole-poor clusters in the ensemble.

\section{CONCLUSIONS}

In summary, our exact Hubbard cluster calculations, at various fillings, show the existence of charge and spin pair- 
ing, electronic phase separation, pseudogaps, and condensation driven by electron correlations. These clusters demonstrate a rich variety of properties which can be tuned by electron or hole doping. Such exact cluster studies may be useful in understanding the phase diagram and superconductivity driven by purely electronic means (such as in CNs). Furthermore, it is quite surprising to see the number of properties that these exact clusters share with the HTSCs. This may be, at least in part, due to the fact that in all these "bad" metallic high $T_{c}$ materials, short-range correlations play a key role.

\section{ACKNOWLEDGMENT}

This research was supported in part by the U.S. Department of Energy under Contract No. DE-AC02-98CH10886.
${ }^{1}$ A. N. Kocharian, G. W. Fernando, K. Palandage, and J. W. Davenport, Phys. Rev. B 74, 024511 (2006).

${ }^{2}$ A. N. Kocharian, G. W. Fernando, K. Palandage, and J. W. Davenport, J. Magn. Magn. Mater. 300, e585 (2006).

${ }^{3}$ H. Shiba and P. A. Pincus, Phys. Rev. B 5, 1966 (1972).

${ }^{4}$ J. Callaway, D. P. Chen, and R. Tang, Phys. Rev. B 35, 3705 (1987).

${ }^{5}$ Claudius Gros, Phys. Rev. B 53, 6865 (1996).

${ }^{6}$ F. Lopez-Urias and G. M. Pastor, Phys. Rev. B 59, 5223 (1999).

${ }^{7}$ E. Dagotto, Rev. Mod. Phys. 66, 763 (1994).

${ }^{8}$ A. N. Kocharian and Joel H. Sebold, Phys. Rev. B 53, 12804 (1996).

${ }^{9}$ R. Schumann, Ann. Phys. (N.Y.) 11, 49 (2002).

${ }^{10}$ J. E. Hirsch, Phys. Rev. B 67, 035103 (2003).

${ }^{11}$ J. Bonca and P. Prelovsěk, Int. J. Mod. Phys. B 17, 3377 (2003).

${ }^{12}$ A. N. Kocharian, G. W. Fernando, K. Palandage, and J. W. Davenport, cond-mat/0701022.

${ }^{13}$ T. Yildirim, O. Zhou, J. E. Fischer, N. Bykovetz, R. A. Strongin, M. A. Cichy, A. B. Smith III, C. L. Lin, and R. Jelinek, Nature (London) 360, 568 (1992).

${ }^{14}$ A. Kasumov, M. Kociak, M. Ferrier, R. Deblock, S. Gueron, B. Reulet, I. Khodos, O. Stephan, and H. Bouchiat, Phys. Rev. B 68, 214521 (2003).

${ }^{15}$ M. A. Tanatar, T. Ishiguro, S. Kagoshima, N. D. Kushch, and E. B. Yagubskii, Phys. Rev. B 65, 064516 (2002).
${ }^{16}$ S. Belluci, M. Cini, P. Onorato, and E. Perfetto, J. Phys.: Condens. Matter 18, S2115 (2006); W-F. Tsai and S. A. Kivelson, Phys. Rev. B 73, 214510 (2006); S. R. White, S. Chakravarty, M. P. Gelfand, and S. A. Kivelson, ibid. 45, 5062 (1992); R. M. Fye, M. J. Martins, and R. T. Scalettar, ibid. 42, R6809 (1990); N. E. Bickers, D. J. Scalapino, and R. T. Scalettar, Int. J. Mod. Phys. B 1, 687 (1987).

${ }^{17}$ J. M. Tranquada, B. J. Sternlieb, J. D. Axe, Y. Nakamura, and S. Uchida, Nature (London) 375, 561 (1995).

${ }^{18}$ T. Koma and H. Tasaki, Phys. Rev. Lett. 68, 3248 (1992).

${ }^{19}$ P. W. Anderson, Science 235, 1196 (1987).

${ }^{20}$ X. Xu, S. Yin, R. Moro, and W. A. de Heer, Phys. Rev. Lett. 95, 237209 (2005).

${ }^{21}$ I. Baldea, H. Koppel, and L. S. Cederbaum, Eur. Phys. J. B 20, 289 (2001).

${ }^{22}$ G. V. M. Williams, J. L. Tallon, and J. W. Loram, Phys. Rev. B 58, 15053 (1998).

${ }^{23}$ Xiao-Jia Chen, Viktor V. Struzhkin, Russell J. Hemley, Ho-kwang Mao, and Chris Kendziora, Phys. Rev. B 70, 214502 (2004).

${ }^{24}$ H. E. Mohottala, B. O. Wells, J. I. Budnick, W. A. Hines, C. Niedermayer, L. Udby, C. Bernard, A. R. Moodenbaugh, and Fang-Cheng Chou, Nat. Mater. 5, 377 (2006).

${ }^{25}$ Y. Itoh, M. Matsumara, and H. Yamagata, J. Phys. Soc. Jpn. 66, 3383 (1997). 